\title{
Trombosis venosa cerebral y COVID-19: ¿Asesino silencioso durante la pandemia?
}

\author{
Cerebral venous thrombosis and COVID-19: A silent killer during the pandemic? \\ Juan Diego Gutiérrez-Zevallos 1,a,b, Diego Alberto Guarniz-Huamán ${ }^{1, a, c,}$, Manuel Sánchez-Landers 1,2,d,e,,g
}

\section{RESUMEN}

Trombosis venosa cerebral (TVC) es una rara condición clínica caracterizada por una obstrucción de los senos venosos durales o de las venas corticales cerebrales que desencadena hipertensión intracraneal y síntomas tales como cefalea, crisis epilépticas y coma, entre otros. Se ha asociado como complicación neurológica del síndrome respiratorio agudo severo (SARS-CoV-2) del Coronavirus 2. Objetivo: Describir los casos reportados de TVC en pacientes con Enfermedad por Coronavirus 2019 (COVID-19). Material y Métodos: Se realizó una búsqueda bibliográfica en PubMed para recabar sólo los reportes de caso en los que los pacientes con COVID-19 desarrollaron TVC. Resultados: Se analizaron reportes de 13 pacientes y se recabó información sobre sexo, edad, comorbilidades, severidad del COVID-19, tipo de TVC, tratamiento recibido, complicaciones y desenlace final. Conclusión: TVC asociada a COVID-19 se puede presentar en pacientes con características variadas, suele tener complicaciones graves y en algunos casos, un desenlace mortal.

PALABRAS CLAVE: Trombosis de los senos intracraneales, trombosis de la vena, venas cerebrales, COVID-19, SARS-CoV-2.

\section{SUMMARY}

Cerebral venous thrombosis (CVT) is a rare clinical condition that consists of an obstruction of the dural venous sinuses or the cerebral cortical veins, triggering intracranial hypertension and symptoms such as headache, seizures and coma among others. CVT has recently been associated as a neurological complication of the Severe Acute Respiratory Syndrome coronavirus 2 (SARS-CoV-2). Objective: To describe reported cases of CVT in patients with Coronavirus Disease 2019 (COVID - 19). Material and Methods: A literature search was conducted on PubMed to collect only case reports of patients with COVID-19 who developed CVT. Results: Report of 13 patients were analyzed and information was collected about sex, age, comorbidities, severity of COVID-19, type of CVT, treatments received, complications and the final outcome. Conclusion: CVT associated with COVID-19 can occur in patients with varied characteristics, often with serious complications and in some cases, a fatal outcome.

KEYWORDS: Intracranial sinus thrombosis, venous thrombosis, cerebral veins, COVID-19, SARS-CoV-2.

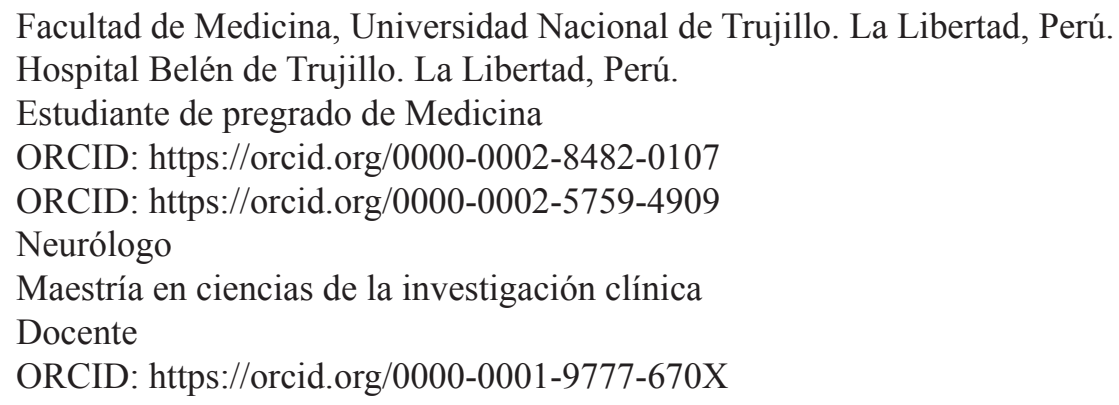




\section{INTRODUCCIÓN}

La COVID-19 es, actualmente, el foco de atención de las instituciones sanitarias a nivel mundial, ya que, a la fecha de este estudio, ha cobrado la vida de casi un millón y medio de personas y se han notificado más de cincuenta millones de casos confirmados (1). Esta nueva enfermedad tiene un amplio espectro de presentaciones, que va desde una infección asintomática, hasta la rápida disfunción multiorgánica y muerte, donde el principal componente afectado es el aparato respiratorio; no obstante, recientemente se han descrito complicaciones neurológicas con mayor frecuencia (2-4). La asociación entre la COVID-19 y las enfermedades cerebrovasculares (ECV) supone un importante campo de estudio para el personal de salud, ya que en el contexto de la infección por el SARS$\mathrm{CoV}-2$ son comunes las alteraciones en los parámetros de coagulación, lo que podría desencadenar una serie de complicaciones trombóticas arteriales y venosas, dentro de ellas, la trombosis venosa cerebral (TVC) (5).

La TVC es una enfermedad poco frecuente que corresponde a una localización anatómica específica de una trombosis venosa típica. Afecta predominantemente a mujeres jóvenes, tiene factores de riesgo como: embarazos múltiples, tromboembolismo venoso, terapia con anticonceptivos orales, entre otros; y cuenta con una incidencia de entre 1 a 1,5 / 100000 personas por año (6-9). La TVC consiste en la obstrucción de los senos venosos o venas cerebrales por un trombo. Esto da lugar al aumento de la presión intracraneal, manifestándose la sintomatología principal de los pacientes con TVC: cefalea subaguda $(90 \%)$, crisis epilépticas (40\%), focalizaciones $(50 \%)$ y en algunos casos, coma (10 $\%)(7,9)$. Debe sospecharse el diagnóstico de TVC en los pacientes con cefalea progresiva en el contexto de los factores de riesgo ya mencionados. Como prueba confirmatoria se prefiere la venografía por tomografía computarizada en combinación con la tomografía simple de cabeza, que tienen una precisión de hasta el $90-100 \%(7,10)$. Para el tratamiento agudo se recomienda la anticoagulación precoz, utilizando heparina de bajo peso molecular vía subcutánea (10). Además, se debe manejar la hipertensión intracraneal, las crisis epilépticas, y las otras manifestaciones con las que se presente el paciente (7).

Las enfermedades cerebrovasculares (ECV) agudas en general, tienen una prevalencia del 0.5 -
5.9\% en pacientes con COVID-19 y si nos referimos a la TVC, Favas et al. encontraron una prevalencia del $0,3 \%$ en pacientes infectados con SARS-CoV-2 (11). El objetivo de este trabajo fue describir los casos reportados de trombosis venosa cerebral en pacientes con COVID-19.

\section{MATERIAL Y MÉTODOS}

Se hizo una búsqueda exhaustiva de la literatura en la base de datos electrónica PubMed. Los criterios de inclusión fueron estudios tipo reportes de caso, pacientes de cualquier edad y con cualquier tipo de trombosis venosa cerebral que se haya presentado concomitantemente con COVID-19. Se obtuvieron todos los estudios comprendidos desde 1 de diciembre de 2019 hasta la fecha de recopilación (30 de octubre de 2020). Se realizaron las búsquedas en PubMed usando los términos MeSH para vincular el concepto de trombosis venosa cerebral con el concepto de COVID-19: "Intracranial Thrombosis"[Mesh] AND ("COVID-19" [Supplementary Concept]) AND 2019/12/01:2020/10/30[Date - Entry]. Para identificar las publicaciones más recientes a las que aún no se les asignaron términos $\mathrm{MeSH}$, la búsqueda se complementó con palabras de texto libre en PubMed: ("Cerebral Vein Thrombosis"[All Fields] OR "Cerebral venous sinus thrombosis"[All Fields] OR "Cerebral venous thrombosis"[All Fields]) AND ("COVID-19"[All Fields] OR "SARS-CoV-2"[All Fields]) AND 2019/12/01:2020/10/31[Date - Entry]. La búsqueda se limitó a publicaciones relacionadas con humanos y en los idiomas inglés, francés y español. La verificación de los criterios de inclusión fue realizada independientemente por los autores y se excluyó aquellos estudios que no cumplían los criterios.

\section{RESULTADOS}

Se analizó un total de 10 reportes de caso usando la fórmula de búsqueda en PubMed luego de haberse excluido los estudios repetidos. Algunos estudios contenían más de un paciente, por lo que el número de casos totales fue de 13. Los hallazgos de los casos publicados se resumen en la Tabla 1. De todos los casos revisados, solo una paciente de sexo femenino presentó, como factor de riesgo para TVC, el uso de anticonceptivos orales (ACO) (12). Un hombre de 63 años presentó diabetes y asma controlados (13). Una mujer de 72 años presentó hipertensión arterial, diabetes y asma controlados (14). En el resto 
Trombosis venosa cerebral y COVID-19: ¿Asesino silencioso durante la pandemia?

Tabla 1: Reportes de caso de pacientes con trombosis venosa cerebral y COVID-19.

\begin{tabular}{|c|c|c|c|c|c|c|c|}
\hline Caso & $\begin{array}{l}\text { Género y } \\
\text { Edad }\end{array}$ & $\begin{array}{l}\text { Severidad de } \\
\text { COVID-19 al } \\
\text { ingreso }\end{array}$ & Cuadro clínico & $\begin{array}{l}\text { Perfil de } \\
\text { coagulación }\end{array}$ & Tipo de TVC & $\begin{array}{l}\text { Tratamiento para } \\
\text { TVC }\end{array}$ & Complicaciones \\
\hline $1(3)$ & $\begin{array}{l}\text { Mujer, } 29 \\
\text { años }\end{array}$ & Leve & $\begin{array}{l}\text { Convulsiones, } \\
\text { cefalea, afasia, } \\
\text { paresia faciobraquial } \\
\text { derecha, tos, fiebre y } \\
\text { disnea. }\end{array}$ & Normal & $\begin{array}{l}\text { Trombosis de } \\
\text { SVD }\end{array}$ & Enoxaparina SC & Infarto hemorrágico $\square$ \\
\hline $2(12)$ & $\begin{array}{l}\text { Hombre, } \\
38 \text { años }\end{array}$ & $\begin{array}{l}\text { Moderado- } \\
\text { severo * }\end{array}$ & $\begin{array}{l}\text { Cefalea, alteración } \\
\text { del estado mental, } \\
\text { diarrea y vómitos. }\end{array}$ & No menciona & $\begin{array}{l}\text { Trombosis de } \\
\text { SVD y VC }\end{array}$ & $\begin{array}{l}\text { Enoxaparina SC } \\
\text { y posteriormente } \\
\text { trombectomía. }\end{array}$ & Edema cerebral + \\
\hline $3(12)$ & $\begin{array}{l}\text { Mujer, } 41 \\
\text { años }\end{array}$ & Leve * & Afasia y confusión. & DD elevado & $\begin{array}{l}\text { Trombosis de } \\
\text { SVD y VC }\end{array}$ & $\begin{array}{l}\text { HNF IV con } \\
\text { drenaje ventricular } \\
\text { externo. }\end{array}$ & $\begin{array}{l}\text { Infarto venoso. Hemorragia } \\
\text { intraventricular. } \\
\text { Hidrocefalia } \ddagger\end{array}$ \\
\hline $4(12)$ & $\begin{array}{l}\text { Hombre, } \\
23 \text { años }\end{array}$ & $\begin{array}{l}\text { Moderado- } \\
\text { severo* }\end{array}$ & Cefalea, fiebre, tos. & $\begin{array}{l}\text { DD y Fg } \\
\text { elevados }\end{array}$ & $\begin{array}{l}\text { Trombosis de } \\
\text { SVD y VC }\end{array}$ & No menciona & Infarto hemorrágico + \\
\hline $5(16)$ & $\begin{array}{l}\text { Mujer, } 2 \\
\text { años y } 7 \\
\text { meses }\end{array}$ & $\begin{array}{l}\text { Moderado- } \\
\text { severo * }\end{array}$ & $\begin{array}{l}\text { Letargia, hiporexia, } \\
\text { adenopatías } \\
\text { cervicales y } \\
\text { hemiparesia } \\
\text { izquierda. }\end{array}$ & $\begin{array}{l}\text { INR, TP, Fg y } \\
\text { DD elevados }\end{array}$ & $\begin{array}{l}\text { Trombosis de } \\
\text { SVD }\end{array}$ & $\begin{array}{l}\text { Aspirina con } \\
\text { drenaje ventricular } \\
\text { externo }\end{array}$ & $\begin{array}{l}\text { Deterioro del estado } \\
\text { respiratorio } \square\end{array}$ \\
\hline $6(17)$ & $\begin{array}{l}\text { Hombre, } \\
56 \text { años }\end{array}$ & $\begin{array}{l}\text { Moderado- } \\
\text { severo }\end{array}$ & $\begin{array}{l}\text { Fiebre y malestar } \\
\text { general. Cefalea y } \\
\text { vómitos en el día } \\
12 .\end{array}$ & $\begin{array}{l}\text { DD y ferritina } \\
\text { elevados }\end{array}$ & $\begin{array}{l}\text { Trombosis de } \\
\text { SVD }\end{array}$ & HNF IV & TEP $\square$ \\
\hline $7(2)$ & $\begin{array}{l}\text { Hombre, } \\
65 \text { años }\end{array}$ & Moderado & $\begin{array}{l}\text { Pérdida del } \\
\text { conocimiento, } \\
\text { oftalmoparesia. }\end{array}$ & No menciona & $\begin{array}{l}\text { Trombosis de } \\
\text { SVD }\end{array}$ & $\begin{array}{l}\text { Anticoagulantes + } \\
\text { levetiracetam }\end{array}$ & Infarto hemorrágico $\square$ \\
\hline $8(13)$ & $\begin{array}{l}\text { Hombre, } \\
63 \text { años }\end{array}$ & Moderado & $\begin{array}{l}\text { Hemiplejía } \\
\text { izquierda con } \\
\text { Babinski. Fiebre, } \\
\text { tos y disnea semana } \\
\text { previa. }\end{array}$ & $\begin{array}{l}\text { DD y Fg } \\
\text { elevados }\end{array}$ & $\begin{array}{l}\text { Trombosis de } \\
\text { SVD }\end{array}$ & Enoxaparina SC & $\begin{array}{l}\text { Infartos venosos y } \\
\text { hemorragia cortical } \\
\text { aguda. Estado epiléptico } \\
\text { convulsivo } \square\end{array}$ \\
\hline $9(14)$ & $\begin{array}{l}\text { Hombre, } \\
51 \text { años }\end{array}$ & Severo* & $\begin{array}{l}\text { Fiebre, tos y disnea } \\
\text { con IOT + VM. } \\
\text { Después de retirar } \\
\text { sedación, coma con } \\
\text { pupilas midriáticas } \\
\text { arreactivas. }\end{array}$ & No menciona & $\begin{array}{l}\text { Trombosis de } \\
\text { SVD }\end{array}$ & Ninguno & $\begin{array}{l}\text { Miocarditis y lesión renal } \\
\text { aguda. Infarto venoso } \\
\text { hemorrágico y herniación } \\
\text { cerebelosa }+\end{array}$ \\
\hline $10(14)$ & $\begin{array}{l}\text { Mujer, } 72 \\
\text { años }\end{array}$ & $\begin{array}{l}\text { Moderado- } \\
\text { severo* }\end{array}$ & $\begin{array}{l}\text { Disnea, hipoxia, } \\
\text { linfopenia. }\end{array}$ & No menciona & $\begin{array}{l}\text { Trombosis de } \\
\text { SVD }\end{array}$ & Ninguno & $\begin{array}{l}\text { Infarto hemorrágico, } \\
\text { herniación subfalciana y } \\
\text { cerebelosa } t\end{array}$ \\
\hline $11(18)$ & $\begin{array}{l}\text { Hombre, } \\
72 \text { años }\end{array}$ & $\begin{array}{l}\text { Moderado- } \\
\text { severo* }\end{array}$ & $\begin{array}{l}\text { Hemiparesia } \\
\text { izquierda, estado } \\
\text { mental alterado, } \\
\text { estado epiléptico } \\
\text { refractario. } \\
\text { Síntomas } \\
\text { respiratorios días } \\
\text { previos. }\end{array}$ & No menciona & $\begin{array}{l}\text { Trombosis de } \\
\text { SVD y VC }\end{array}$ & Anticoagulación & $\begin{array}{l}\text { Infarto venoso } \\
\text { hemorrágico }\end{array}$ \\
\hline $12(19)$ & $\begin{array}{l}\text { Mujer, } 63 \\
\text { años }\end{array}$ & Leve & $\begin{array}{l}\text { Afasia y hemiplejía } \\
\text { derecha. Fiebre, tos } \\
\text { y anosmia } 12 \text { días } \\
\text { previos. }\end{array}$ & Fg elevado & $\begin{array}{l}\text { Trombosis de } \\
\text { SVD }\end{array}$ & Enoxaparina SC & $\begin{array}{l}\text { Hemorragia cerebral. } \\
\text { Estatus epiléptico } \$\end{array}$ \\
\hline $13(15)$ & $\begin{array}{l}\text { Hombre, } \\
30 \text { años }\end{array}$ & Leve & $\begin{array}{l}\text { Crisis tónicas } \\
\text { generalizadas. } \\
\text { COVID-19 } \\
\text { asintomático. }\end{array}$ & $\begin{array}{l}\text { TP y DD } \\
\text { elevados }\end{array}$ & $\begin{array}{l}\text { Trombosis de } \\
\text { SVD y vena } \\
\text { yugular interna }\end{array}$ & $\begin{array}{l}\text { Enoxaparina SC y } \\
\text { luego rivaroxabán }\end{array}$ & $\begin{array}{l}\text { Ictus isquémico pequeño y } \\
\text { hemorragia subaracnoidea } \\
\square\end{array}$ \\
\hline
\end{tabular}

TP, tiempo de protrombina; DD, dímero D; Fg, fibrinógeno; TVC, trombosis venosa cerebral; SVD, senos venosos durales; VC, venas cerebrales; IOT, intubación orotraqueal; VM, ventilación mecánica; TEP, tromboembolismo pulmonar; SC, subcutánea; HNF, heparina no fraccionada; IV, intravenosa *Severidad del COVID-19 empeoró y paciente necesito intubación orotraqueal y ventilación mecánica.

$\square$ Mejoría clínica

$\ddagger$ Muerte 
de pacientes $(84,6 \%)$ no se identificaron factores de riesgo significativos antes del episodio de TVC. El $61,5 \%$ de los casos fueron hombres. La edad promedio fue $46,5 \pm 21,3$ años. 53,8\% presentó anormalidades del perfil de coagulación como dímero D $(46,1 \%)$, fibrinógeno, tiempo de protrombina o INR elevados. Solo un paciente tuvo el perfil de coagulación normal y en los demás no se mencionó esa información. Ninguna paciente se encontraba embarazada o en puerperio. Solo un paciente $(7,6 \%)$ no tuvo ningún síntoma adicional relacionado a COVID-19 (15). $23 \%$ de pacientes se mantuvieron como casos leves y un 53,8\% necesitó ventilación mecánica. Todos los pacientes tuvieron afectación de senos venosos durales, 30,7\% tuvo afectación en venas cerebrales y un paciente tuvo trombosis en vena yugular interna. $53,8 \%$ de los casos encontrados tuvieron un desenlace mortal.

\section{DISCUSIÓN}

La TVC es una condición neurológica potencialmente mortal, que con tratamiento oportuno se reduce bastante la morbimortalidad, pero que en muchas ocasiones no se diagnostica a tiempo, lo que ensombrece su pronóstico (20). Esta rápida revisión de reportes de caso ofrece una descripción general de los pacientes con COVID-19 que desarrollaron TVC. Nuestros principales hallazgos son que la TVC se puede presentar como única manifestación de COVID-19 o incluso en casos leves de esta nueva enfermedad. Además, debido a que los pacientes que necesitan ventilación mecánica tienen que estar sedados durante semanas, solamente después de retirar sedación endovenosa nos enteramos de la existencia de complicaciones neurológicas. Esto fue frecuente en los reportes de caso que hallamos, y el diagnóstico tardío se asociaba a pacientes en coma o con muerte cerebral.

Las afecciones vasculares en el SNC relacionadas al COVID-19 no son exclusivas de adultos mayores con factores de riesgo cardiovasculares (hipertensión arterial y diabetes mellitus); sin embargo, la presencia de éstos sí se asocian a COVID-19 severo con una respuesta inflamatoria incrementada y estado hipercoagulable que subyace a la TVC (21). Esto explica el porqué muchos pacientes no eran jóvenes como es usual en TVC (37 años en promedio) (22). Tampoco concuerda que la mayoría de casos hayan sido hombres ya que TVC suele afectar más a mujeres en ratio mujer - varón de 3:1 (10), aunque esto puede ser un sesgo por la poca cantidad de casos.
Debido a la presencia de receptores ACE2 en el cerebro, se sugiere que el SARS-CoV-2 invade el endotelio vascular, lo daña y provoca agregación plaquetaria. Además, la alteración de la coagulación producto de la tormenta de citocinas mediante una respuesta inflamatoria altera tanto plaquetas, como aumento de dímero D y fibrinógeno (16). La evidencia del mecanismo exacto de este estado hipercoagulable se sigue formando, pero la respuesta inflamatoria y la inmovilización prolongada de los pacientes con COVID-19 podrían ayudar a explicar la formación de TVC (14).

Tu et al también revisó reportes de caso y encontró valores concordantes con nuestros resultados como edad promedio de 43 años, ligera predominancia del sexo masculino, $33,3 \%$ de casos de trombosis en venas cerebrales, ausencia de factores de riesgo significativos en el $57,1 \%$ de casos, $14,3 \%$ de pacientes sin síntomas relacionados a COVID-19 y 37\% de casos leves de infección por SARS-CoV-2 (23). Ellos encontraron más casos de dímero D elevado (75\%) y que TVC afectaba casi equitativamente a los diversos grados de severidad de COVID-19. Fraiman et al reporta que de 14 casos de TVC en pacientes COVID-19, el 50\% fueron varones, edad promedio fue de 49 años, $50 \%$ no tenía factores de riesgo para TVC, $57,1 \%$ tuvo dímero D elevado, 76,9\% tuvo afectación en senos venosos, $23 \%$ en la vena de Galeno, y un $64,2 \%$ tuvo infarto venoso con transformación hemorrágica (24). Por su parte, Nwajei et al refiere una mortalidad del $36,4 \%$, predominancia del sexo femenino $(54,5 \%)$ y edades entre 29 y 72 años (25).

En casi todos los casos reportados se optó por el tratamiento anticoagulante como primera elección para tratar la TVC, esto concuerda con las directrices de tratamiento del tromboembolismo venoso en pacientes con COVID-19, las cuales también se aplicaron para TVC con buenos resultados (17). Esta guía recomienda la anticoagulación parenteral con heparina de bajo peso molecular sobre la terapia no anticoagulante; además, alerta sobre el riesgo del uso de tratamiento antiplaquetario y con anticoagulantes orales directos (26).

Los casos reportados de TVC en pacientes con COVID-19 demuestran que esta complicación neurológica se puede dar en pacientes de edades variadas, incluso en ausencia de comorbilidades. Estos pacientes suelen tener complicaciones graves y en algunos casos un desenlace mortal, por lo que el personal de salud debe tener conocimiento sobre el riesgo potencial de la TVC en este contexto. 
Declaración de Financiamiento: Autofinanciamiento Conflictos de Intereses: Sin conflicto de interés

\section{Correspondencia:}

Juan Diego Gutierrez-Zevallos

Pueblo Joven El Bosque Mz C Lote 28. Trujillo. La Libertad. Perú.

Código postal: 13007

Correo electrónico: jgutierrezz@unitru.edu.pe

\section{REFERENCIAS BIBLIOGRÁFICAS}

1. World Health Organization. WHO Coronavirus Disease (COVID-19) Dashboard. Ginebra: orld Health Organization; 2020. (Citado 10 de noviembre de 2020). Disponible en: https://covid19.who.int/

2. Hemasian H, Ansari B. First case of Covid-19 presented with cerebral venous thrombosis: A rare and dreaded case. Rev Neurol (Paris). 2020;176(6):521-3.

3. Klein DE, Libman R, Kirsch C, Arora R. Cerebral venous thrombosis: A typical presentation of COVID-19 in the young. J Stroke Cerebrovasc Dis. 2020; 29(8): 104989.doi: $10.1016 / j$. jstrokecerebrovasdis.2020.104989

4. Kananeh MF, Thomas T, Sharma K, Herpich F, Urtecho $\mathrm{J}$, Athar MK, et al. Arterial and venous strokes in the setting of COVID-19. J Clin Neurosci. 2020;79:60-6.

5. Dahl-Cruz F, Guevara-Dalrymple N, LópezHernández N. Trombosis venosa cerebral e infección por SARS-CoV-2. Rev Neurol. 2020;70(10):391-2.

6. Silvis SM, De-Sousa DA, Ferro JM, Coutinho JM. Cerebral venous thrombosis. Nat Rev Neurol. 2017;13(9):555-65.

7. Zuurbier SM, Coutinho JM. Cerebral venous thrombosis. Adv Exp Med Biol. 2017; 906:183-93.

8. Ferro JM, Aguiar-de-Sousa D. Cerebral Venous Thrombosis: an Update. Curr Neurol Neurosci Rep. 2019;19(10):74

9. Kristoffersen ES, Harper CE, Vetvik KG, Faiz KW. Cerebral venetrombose - Forekomst, diagnostikk og behandling. Tidsskr den Nor Laegeforening. 2018;138(12). doi: 10.4045/tidsskr.17.1047

10. Behrouzi R, Punter M. Diagnosis and management of cerebral venous thrombosis. Clin Med J R Coll Physicians London. 2018;18(1):75-9.

11. Favas TT, Dev P, Chaurasia RN, Chakravarty K, Mishra R, Joshi D, et al. Neurological manifestations of COVID-19: a systematic review and meta-analysis of proportions. Neurol Sci. 2020;41:3437-70.

12. Cavalcanti DD, Raz E, Shapiro M, Dehkharghani S, Yaghi S, Lillemoe $\mathrm{K}$, et al. Cerebral venous thrombosis associated with COVID-19. AJNR Am J
Neuroradiol. 2020;41(8):1370-1376. doi: 10.3174/ ajnr.A6644

13. Bolaji P, Kukoyi B, Ahmad N, Wharton C. Extensive cerebral venous sinus thrombosis: A potential complication in a patient with COVID-19 disease. BMJ Case Rep. 2020;13(8):13-6.

14. Keaney K, Mumtaz T. Cerebral venous thrombosis in patients with severe COVID-19 infection in intensive care. Br J Hosp Med (Lond). 2020;81(9):1-4.

15. Hussain S, Vattoth S, Haroon KH, Muhammad A. A Case of Coronavirus Disease 2019 presenting with seizures secondary to cerebral venous sinus thrombosis. Case Rep Neurol. 2020;12(2):260-5.

16. Essajee F, Solomons R, Goussard P, Van-Toorn R. Child with tuberculous meningitis and COVID-19 coinfection complicated by extensive cerebral sinus venous thrombosis. BMJ Case Rep. 2020;13(9):1-3.

17. Sugiyama Y, Tsuchiya T, Tanaka R, Ouchi A, Motoyama A, Takamoto T, et al. Cerebral venous thrombosis in COVID-19-associated coagulopathy: A case report. J Clin Neurosci. 2020;79:30-2.

18. Chougar L, Mathon B, Weiss N, Degos V, Shor N. Atypical Deep Cerebral Vein Thrombosis with Hemorrhagic Venous Infarction in a Patient Positive for COVID-19. Am J Neuroradiol. 2020;41(8):1377-9.

19. Roy-Gash F, Marine DM, Jean-Michel D, Herve V, Raphael B, Nicolas E. COVID-19-associated acute cerebral venous thrombosis: Clinical, CT, MRI and EEG features. Crit Care. 2020;24(1):1-3.

20. Idiculla PS, Gurala D, Palanisamy M, Vijayakumar R, Dhandapani S, Nagarajan E. Cerebral venous thrombosis: A comprehensive review. Eur Neurol. 2020;83(4):369-79.

21. Li Y, Li M, Wang M, Zhou Y, Chang J, Xian Y, et al. Acute cerebrovascular disease following COVID-19: a single center, retrospective, observational study. Stroke Vasc Neurol. 2020;5(3):279-84.

22. Rebolledo-García D, Domínguez-Moreno R, González-Vargas P. Trombosis venosa cerebral: lo que hay que saber. Med Int Méx. 2019;35(4):537-52.

23. Tu TM, Goh C, Tan YK, Leow AS, Pang YZ, Chien J, et al. Cerebral Venous Thrombosis in Patients with COVID-19 Infection: a Case Series and Systematic Review. J Stroke Cerebrovasc Dis. 2020;29(12): 105379. doi: 10.1016/j.jstrokecerebrovasdis. 2020.105379

24. Fraiman P, Godeiro Junior C, Moro E, Cavallieri F, Zedde M. COVID-19 and Cerebrovascular Diseases: A Systematic Review and Perspectives for Stroke Management. Front Neurol. 2020;11:574694. doi: 10.3389/fneur.2020.574694

25. Nwajei F, Anand P, Abdalkader M, Andreu Arasa VC, Aparicio HJ, Behbahani S, et al. Cerebral Venous Sinus Thromboses in Patients with SARS-CoV-2 
Infection: Three Cases and a Review of the Literature. J Stroke Cerebrovasc Dis. 2020;29(12):105412. doi: 10.1016/j.jstrokecerebrovasdis.2020.105412

26. Moores LK, Tritschler T, Brosnahan S, Carrier M, Collen JF, Doerschug K, et al. Prevention, Diagnosis, and Treatment of VTE in Patients With Coronavirus Disease 2019: CHEST Guideline and Expert Panel Report. Chest. 2020;158(3):1143-63.

Recibido: $13 / 11 / 2020$ Aceptado: 02/12/2020 